\title{
Gaussian Process modelling of blood glucose response to free-living physical activity data in people with type 1 diabetes
}

\author{
John Joseph Valletta, Student Member, IEEE, Andrew J. Chipperfield and Christopher D. Byrne
}

\begin{abstract}
Good blood glucose control is important to people with type 1 diabetes to prevent diabetes-related complications. Too much blood glucose (hyperglycaemia) causes long-term micro-vascular complications, while a severe drop in blood glucose (hypoglycaemia) can cause life-threatening coma. Finding the right balance between quantity and type of food intake, physical activity levels and insulin dosage, is a daily challenge. Increased physical activity levels often cause changes in blood glucose due to increased glucose uptake into tissues such as muscle. To date we have limited knowledge about the minute by minute effects of exercise on blood glucose levels, in part due to the difficulty in measuring glucose and physical activity levels continuously, in a free-living environment. By using a light and user-friendly armband we can record physical activity energy expenditure on a minute-by-minute basis. Simultaneously, by using a continuous glucose monitoring system we can record glucose concentrations. In this paper, Gaussian Processes are used to model the glucose excursions in response to physical activity data, to study its effect on glycaemic control.
\end{abstract}

\section{INTRODUCTION}

Maintaining normoglycaemia is a daily challenge for people with type 1 diabetes. Timely administration of insulin and a healthy diet are vital in keeping blood glucose within the physiologically acceptable range of $4-7 \mathrm{mmol} / \mathrm{l}$. Extensive research has been going on in the last thirty years in modelling the glucose-insulin system [1]. Most of the models proposed are based on either the Bergman's minimal model [2] or Sorensen's physiological model [3]. These models offer a qualitative prediction tool for blood glucose, in response to exogenous insulin and carbohydrate intake. It was only recently that [4] and [5] extended Bergman's and Sorensen's model respectively to account for the effect of physical activity. Exercise has major effects on blood glucose, mainly due to the increase of glucose uptake in target peripheral tissues such as muscle. In both cases this was modelled by a single parameter $P V 0_{2}^{\max }$, which represents the percentage of the maximal oxygen consumption $\left(\mathrm{VO}_{2}^{\max }\right)$. Both models are physiologically based and can explain qualitatively what happens during varying levels of physical activity. However, they are not data-true models and hence cannot fully explain

This study is being supported by Diabetes UK under grant BDA: RD06/0003306

J.J. Valletta and A.J. Chipperfield are with the School of Engineering Sciences, Computational Engineering and Design Group, University of Southampton, Southampton, United Kingdom jj.vallettaesoton.ac.uk and a.j.chipperfieldesoton.ac.uk

C.D. Byrne is with the School of Medicine, Development Origins of Health and Disease, Endocrinology and Metabolism Unit, Southampton General Hospital, Southampton, United Kingdom c.d.byrne@soton.ac.uk the erratic changes in blood glucose which occur normally in people with type 1 diabetes.

Rollins et al. [6] and Vyas et al. [7] were the first to model free-living physical activity data from the armband [8] and relate it to blood glucose. In both cases the data was obtained from a single type 2 diabetic wearing the armband and the continuous glucose monitoring system (CGMS) for 25 days. They used Wiener models and statistical machine learning algorithms respectively to identify data-true models which explain the variation in blood glucose levels, given the physiological signals measured by the armband.

In this paper, Gaussian Processes are used to obtain datatrue models for data coming from type 1 diabetic people wearing both the armband and the CMGS at the same time. Being completely insulin deficient, type 1 diabetics exhibit a more pronounced excursion from the normoglycaemic range compared to type 2 . This makes the modelling process even more challenging.

\section{METHODS AND DATA ACQUISITION}

In this section, after briefly presenting the study protocol, the two main data acquisition devices used in the study are discussed.

\section{A. Study Protocol}

This study was approved by the local ethics committee LREC: 07/H0502/134. So far a total of 18 people (9 females) have been recruited in this ongoing study. Table I shows the characteristic data for this cohort, displayed as mean \pm standard deviation. After consenting to the study, several clinical baseline tests are performed to establish individual physiological parameters. These have been deemed to be beyond the scope of this paper discussion and hence omitted. Each participant was then given a SenseWear ${ }^{\circledR}$ Pro 2 armband [8] and the Guardian ${ }^{\circledR}$ CGMS [9]. These were worn for approximately two weeks (the glucose sensor needs to be replaced every three days). At the same time the participant was asked to keep a detailed food and insulin diary. This data collection is repeated again after six months to study seasonal variations in glycaemic control.

\section{B. SenseWear ${ }^{\circledR}$ armband}

The armband monitors physical activity using five different sensors:

1) Transversal acceleration (measure of movement)

2) Longitudinal acceleration (measure of movement)

3) Heat flux (average heat dissipated or absorbed by the arm) 
TABLE I

PARTICIPANT'S BASELINE CHARACTERISTICS

\begin{tabular}{|l||l|}
\hline Characteristic & Value \\
& $\mathrm{n}=18,9$ Females \\
\hline Age & $36.9 \pm 11.6$ years \\
Years with diabetes & $16.3 \pm 10.9$ years \\
Weight & $73 \pm 14.4 \mathrm{~kg}$ \\
BMI & $25.4 \pm 5.6 \mathrm{~kg} / \mathrm{m}^{2}$ \\
$\mathrm{HbA}_{1 \mathrm{c}}$ & $8.2 \pm 1.6 \%$ \\
\hline
\end{tabular}

4) Galvanic skin response (electrical conductivity between two points on the arm)

5) Skin and near-body temperature

The sensors are sampled at $32 \mathrm{~Hz}$ and an average or MAD (mean of absolute differences) value is stored every minute. These physiological signals are then combined together using a proprietary algorithm to estimate the physical activity energy expenditure.

\section{Medtronic Guardian ${ }^{\circledR}$ Real-Time Continuous Glucose Monitoring System}

To understand the minute-by-minute relationship between exercise and blood glucose, the latter needs to be continuously monitored. The Guardian ${ }^{\circledR}$ Real-Time Continuous Glucose Monitoring System (CGMS) by Medtronic (MiniMed Inc., CA) is one of the most advanced glucose monitors currently on the market. It uses a needle-type glucose sensor which is inserted subcutaneously and measures interstitial glucose. From this data, blood glucose can be estimated. To avoid measurement noise from causing sudden changes in the read-out, the signal is passed through a rate-limiting filter. Subsequently, the blood glucose estimate is updated every 5 mins. Note that the device needs to be calibrated against a standard finger-prick measurement every 12 hours.

\section{MODELLING FREE-LIVING DATA}

Physiologically-based compartmental models use first order differential equations to describe glucose dynamics. Although they can predict qualitatively the overall effect on blood glucose, they are unable to fit free-living data. Assuming that our prior knowledge is limited, Gaussian Processes [10] will be used to identify data-true black box models. Before doing so, the modelling of insulin and carbohydrate intake is briefly discussed.

\section{A. Modelling Insulin}

Type I diabetics are completely insulin deficient, hence all of the circulating insulin is provided exogenously through subcutaneous injections. Several insulin flow models have been proposed in the literature [11]. The pharmacokinetic model recently proposed by Tarin et al. [12] was employed here since it is a generic model and covers most of the insulin preparations currently available. This model uses three coupled partial differential equations which describe the time-evolution of insulin in both its hexameric and dimeric form. For further details about this generic model, the reader is directed to Tarin et al. [12].

\section{B. Modelling Food Intake}

The digestive system is very complex and only few models have been proposed in the literature. Here we use one of the simplest, yet most popular model, proposed by Lehmann et al. [13]. The amount of glucose entering the bloodstream via the guts is modelled as:

$$
\begin{aligned}
\dot{G}_{\text {gut }}(t) & =G_{\text {empt }}(t)-k_{\text {abs }} G_{\text {gut }}(t) \\
R_{\mathrm{a}}(t) & =k_{\text {abs }} G_{\text {gut }}(t)
\end{aligned}
$$

Where

$G_{\text {gut }}(t)$ is the amount of glucose in the gut $[\mathrm{mmol}]$

$G_{\text {empt }}(t)$ is the rate of gastric emptying $\left[\mathrm{mmol} \mathrm{hr}^{-1}\right]$

$k_{\text {abs }}$ is the rate constant for gut absorption of glucose $\left[h r^{-1}\right]$ $R_{\mathrm{a}}(t)$ is the rate of glucose appearance in the bloodstream [mmol hr-1]

\section{Gaussian Processes}

Gaussian Processes (GPs) is a relatively new data modelling technique popularised within the machine learning community by Rasmussen and Williams [10]. It returns a non-parametric probabilistic model. A GP is defined as an infinite ensemble of random variables, any finite number of which have a joint normal distribution. It is fully characterised by its mean and covariance function [10]:

$$
\begin{aligned}
f(\mathbf{x}) & \sim \mathrm{GP}\left(m(\mathbf{x}), k\left(\mathbf{x}, \mathbf{x}^{\prime}\right)\right) \\
m(\mathbf{x}) & =\mathrm{E}[f(\mathbf{x})] \\
k\left(\mathbf{x}, \mathbf{x}^{\prime}\right) & =\mathrm{E}\left[(f(\mathbf{x})-m(\mathbf{x}))\left(f\left(\mathbf{x}^{\prime}\right)-m\left(\mathbf{x}^{\prime}\right)\right)\right]
\end{aligned}
$$

Where

$\mathbf{x}$ is a $d$-dimensional input space

$f(\mathbf{x})$ is the process we want to model

$m(\mathbf{x})$ is the mean function

$k\left(\mathbf{x}, \mathbf{x}^{\prime}\right)$ is the covariance function

In GPs we specifically model the correlations between the input space. This is very important when dealing with highly correlated inputs (as is the case here). By quantifying the similarity between all of the training inputs and the testing input, a value for the function output is inferred within a Bayesian framework. The prior in this case is placed on the function itself. This is very different from other Bayesian techniques, where the function class is usually chosen beforehand and the prior is put on its parameters. These are then chosen to fit the data.

Thus, in GPs, the mean and covariance functions convey all of our prior knowledge about the process. Without loss of generality the mean function is usually taken to be zero. However, in our application a more informed approximation would be the blood glucose level corresponding to the individual's $\mathrm{HbA}_{1 \mathrm{c}}$ (a clinical measure of long-term average for blood glucose). The covariance function (the similarity metric) is the most important design parameter as it conveys information about the system such as stationarity and smoothness. One of the most popular stationary covariance function is the squared exponential: 


$$
k_{s e}\left(\mathbf{x}, \mathbf{x}^{\prime}\right)=\sigma_{f}^{2} \exp \left(-\frac{1}{2}\left(\mathbf{x}-\mathbf{x}^{\prime}\right)^{T} L\left(\mathbf{x}-\mathbf{x}^{\prime}\right)\right)
$$

Where

$\sigma_{f}^{2}$ is the function variance

$L=\operatorname{diag}(\mathbf{I})^{-2}$ is the length-scale matrix

Note how the length-scale $l$ dictates how 'far' apart each scalar input $x$ needs to be so as to be deemed correlated. Together with $\sigma_{f}^{2}$, these form the hyperparameters of a GP model. These are typically estimated from the data by minimising the marginal log-likelihood. As discussed in [10], the length-scale $l$ can be used for Automatic Relevance Detection (ARD). An input with a very large $l$ will have no effect whatsoever on the inferred output. Hence, that input can be considered to be insignificant. Using this technique, the most important regressors can be chosen.

\section{RESULTS AND DISCUSSIONS}

As an example of a subject-specific model, data collected from a participant is presented in this section. The training data consisted of 6 days, while the validation set consisted of approximately 3 days.

\section{A. Choosing the regressors}

Although ARD can be used to choose the most important regressors, we still need to decide which ones to start with. The more regressors in the input space, the more complex the marginal log-likelihood function becomes and the more local minima it will have. Also, the regressors that we choose must be easily understood by clinicians if they are going to use such models to help treat their patients. Glucose $(g)$, carbohydrate intake (cho) and insulin $(i)$ are the obvious regressors. But what about the physical activity parameters? Transversal and longitudinal acceleration data is difficult to interpret. However, METs (Metabolic EquivalenT) in $(\mathrm{kcal} / \mathrm{kg} / \mathrm{hr})$, a parameter that can be derived from such data (and other variables measured by the armband), is clinically well known and understood. It is a multiple of the resting metabolic rate, that is, the amount of calories that the body 'burns' to keep itself functioning. Heat flux $(h f)$ in $\left(W / m^{2}\right)$ and skin temperature $(s t)$ in $\left({ }^{\circ} \mathrm{C}\right)$ are two other parameters which are easily understood by clinicians. Galvanic skin response $(\mu$ Siemens $/ m)$, on the other hand, was left out because it is difficult to interpret by a diabetologist and from the data collected it was found to have a very small variance. Hence, it is not a persistently exciting input and thus is not suited for model identification purposes.

\section{B. Results}

Following from the previous discussion, the input space will now look like this:

$$
\begin{aligned}
& {[g(k-1) \ldots g(k-n), \operatorname{cho}(k-1) \ldots \operatorname{cho}(k-n),} \\
& \quad i(k-1) \ldots i(k-n), \operatorname{hf}(k-1) \ldots h f(k-n), \\
& \operatorname{st}(k-1) \ldots s t(k-n), \operatorname{met}(k-1) \ldots \operatorname{met}(k-n)](7)
\end{aligned}
$$

Thus, the next step is to choose a value for $n$, the number of lagged variables. Intuitively, lags of $n=6$ for $g, i$ and cho, $n=24$ for $h f$ and st, and lags between 12 and 48 for met were considered. Since the sample time is $5 \mathrm{mins}$, these correspond to lags of 30mins, 2hours and 1hour to 4hours respectively. These reflect the range of time constants for each variable which were verified by simulations. People with type 1 diabetes tend to suffer from hypoglycaemic events well after they have engaged themselves in strenuous exercise. This is indeed reflected by the range of regressors chosen for MET which is our main metric for physical activity.

The squared exponential covariance function defined in 6 was chosen for our model. Measurement noise was modelled using a covariance function of the type $k_{\text {noise }}\left(\mathbf{x}, \mathbf{x}^{\prime}\right)=\sigma_{n}^{2} I$. Where $\sigma_{n}^{2}$ is the noise variance, which is assumed to be constant throughout the whole process. Hence, the complete stationary covariance function is:

$$
k\left(\mathbf{x}, \mathbf{x}^{\prime}\right)=k_{\text {se }}\left(\mathbf{x}, \mathbf{x}^{\prime}\right)+k_{\text {noise }}\left(\mathbf{x}, \mathbf{x}^{\prime}\right)
$$

The marginal log-likelihood was then minimised and after using ARD, the model shown in Table II was identified. Note that all the input signals (apart from glucose, the output variable), were normalised to be between $[+1,-1]$.

TABLE II

IDENTIFIED MODEL

\begin{tabular}{|c|c||c|c|}
\hline Regressor & Length-Scale & Regressor & Length-Scale \\
\hline$g(k-1)$ & 4.0393 & $g(k-2)$ & 5.5136 \\
cho(k-1) & 0.6528 & $c h o(k-6)$ & 0.2285 \\
$i(k-2)$ & 0.5353 & $i(k-3)$ & 0.4737 \\
$h f(k-4)$ & 0.7004 & $s t(k-1)$ & 0.4228 \\
st $(k-3)$ & 0.2893 & $s t(k-13)$ & 0.7160 \\
met $(k-42)$ & 0.4280 & - & - \\
\hline Hyperparameter & Value & Hyperparameter & Value \\
\hline$\sigma_{f}^{2}$ & 2.3924 & $\sigma_{n}^{2}$ & 0.1050 \\
\hline
\end{tabular}

By iteratively feeding back the posterior mean of the output, 5 (25mins), 12 (1hour) and 48 (4hours) -step ahead predictions on the validation set were performed. These are depicted in Figure 1 together with the model-predicted output (that is, infinite-step ahead prediction).

\section{Discussions}

The results obtained suggest that the identified model can predict glucose in the long-term reasonably well. For the model-predicted output it is interesting to note that although the predicted value was consistently higher than the measured one, the rate of change is quite similar. That is, the model was able to track a decrease/increase in blood glucose. This suggests that the assumption of stationarity is not correct. The mean of our system is changing over time. Note that $\mathrm{HbA}_{1 \mathrm{c}}$ is an average over 120 days, hence assuming this average for a three day validation set is only a crude assumption.

There are various other challenges faced in this modelling problem. Carbohydrate intake and insulin models are merely look-up tables. They do not differentiate between subjects. 
Fig. 1. Multiple-Step Ahead Predictions
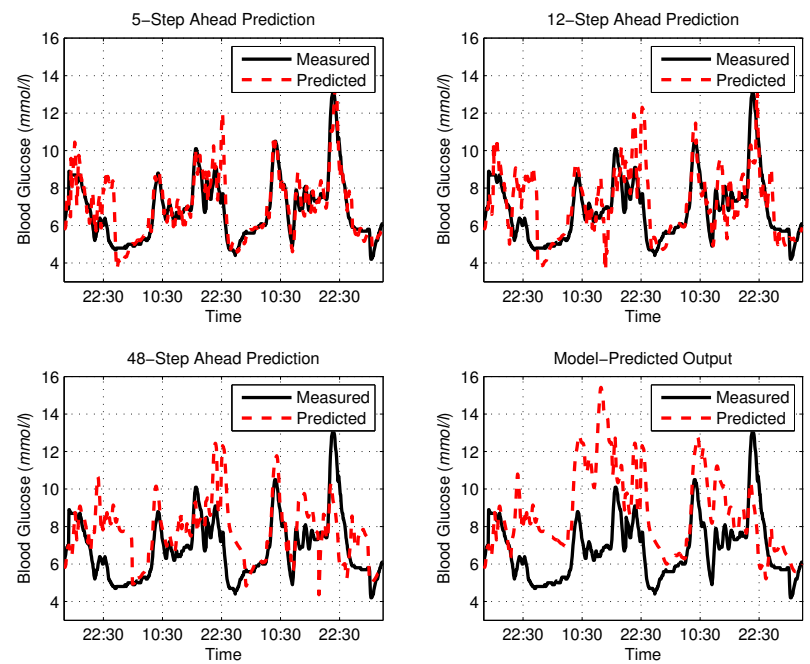

Carbohydrate and insulin absorption vary considerably between people. Also, the assumption that only carbohydrates affect blood sugar is not correct. Fat and protein in food trigger processes (e.g gluconeogenesis) by which glucose is synthesised and supplied to the bloodstream when it is needed. On the other hand insulin absorption depends on various parameters such as the dose, site of application and exercise (which tends to increase the diffusion rate). This information is excluded from the model, which leaves glucose and physical activity as the only measurable variables.

As with any other sensing technology they both suffer from measurement noise. In particular, blood glucose which is estimated indirectly from interstitial glucose. Its accuracy is very dependent on the frequency and quality of the calibrations (at least every 12 hours). Calibrations need to be performed when glucose is the most stable, usually before going to bed, early morning or just before a meal. These calibrations are performed against standard glucose meters, which have an absolute error themselves. As regards the armband, full details of its validation can be found in [8].

Finally, volunteers were normally monitored for up to 12 days (four glucose sensors). Although it would be very useful to prolong this, it is very difficult in practice. Even though the devices used are reasonably small and light, participants do not feel comfortable wearing them for longer. Also, the glucose sensor needs to be replaced every three days (for health and safety reasons). Hence, effectively we are only getting three days of continuous data, because it is very rare that people wear them back-to-back. Considering the large lag between exercise and its effect on blood glucose, this poses another challenge on the modelling problem.

\section{CONCLUSIONS AND FUTURE WORK}

\section{A. Conclusions}

We have shown that Gaussian Processes may provide a good starting point for modelling free-living physical activity data. The importance of these subject-specific models is to be able to understand the lag involved between a person performing daily physical activity and its effect on blood glucose. This information can be related back to an individual's glycaemic control. Specifically, how is the persons lifestyle helping them (or not) in maintaining glucose within a narrow band, both in the short-term and longer-term.

\section{B. Future Work}

We are still in the process of collecting more data from our volunteers. Having obtained data from different people who engage themselves in varying levels of physical activity, we will compare these person-specific models and look for similarities and major differences. A longer-term objective is to identify a single model structure in which parameters are related to different lifestyles. This will enable us to study the benefit, in terms of glycaemic regulation, of changing ones lifestyle.

\section{ACKNOWLEDGMENTS}

The authors gratefully acknowledge Diabetes UK for funding this study under grant BDA: RD06/0003306. Special thanks go to Research Sister Clare Grocott from the Wellcome Trust Clinical Research Facility, Lucinda England and to all the volunteers who are participating in the study.

\section{REFERENCES}

[1] A. Makroglou, J. Li, and Y. Kuang, "Mathematical models and software tools for the glucose-insulin regulatory system and diabetes: an overview," Appl Numer Math, vol. 56, pp. 559-573, 2006.

[2] R. N. Bergman, Y. Z. Ider, C. R. Bowden, and C. Cobelli, "Quantitative estimation of insulin sensitivity," Am J Physiol-Endoc M, vol. 236, no. 6, pp. 667-677, 1979.

[3] J. T. Sorensen, "A physiologic model of glucose metabolism in man and its use to design and assess improved insulin therapies for diabetes," Ph.D. dissertation, Department of Chemical Engineering, MIT, Cambridge, USA, 1985.

[4] A. Roy and R. S. Parker, "Dynamic modeling of exercise effects on plasma glucose and insulin levels," J Diabetes Sci Technol, vol. 1, no. 3, pp. 338-347, 2007.

[5] M. Hernandez-Ordonez and D. Campos-Delgado, "An extension to the compartmental model of type 1 diabetic patients to reproduce exercise periods with glycogen depletion and replenishment," J Biomech, vol. 41, pp. 744-752, 2008.

[6] D. K. Rollins, N. Bhandari, and K. R. Kotz, "Critical modeling issues for successful feedforward control of blood glucose in insulin dependent diabetics," in 2008 American Control Conference, 2008, pp. 832-837.

[7] N. Vyas, D. Andre, R. Jackson, and J. Stivoric, "Development of a non-invasive glucose monitoring system for free-living environments," in Diabetes Technology Society 8th annual meeting, Bethesda, MD2008, 2008.

[8] M. St-Onge, D. Mignault, D. B. Allison, and R. Rabasa-Lhoret, "Evaluation of a portable device to measure daily energy expenditure in free-living adults," Am J Clin Nutr, vol. 85, pp. 742-749, 2007.

[9] N. Sachedina and J. C. Pickup, "Performance assessment of the medtronic-minimed continuous glucose monitoring system and its use for measurement of glycaemic control in type 1 diabetic subjects," Diabetic Med, vol. 20, pp. 1012-1015, 2003.

[10] C. E. Rasmussen and C. K. I. Williams, Gaussian Processes for Machine Learning. Cambridge, Massachusetts: The MIT Press, 2006.

[11] G. Nucci and C. Cobelli, "Models of subcutaneous insulin kinetics. a critical review," Comput Meth Prog Bio, vol. 62, pp. 249-257, 2000.

[12] C. Tarin, E. Teufel, J. Pico, J. Bondia, and H. J. Pfleiderer, "Comprehensive pharmacokinetic model of insulin glargine and other insulin formulations," IEEE T Bio-Med Eng, vol. 52, no. 12, pp. 1994-2005, 2005.

[13] E. D. Lehmann and T. Deutsch, "A physiological model of glucoseinsulin interaction in type 1 diabetes mellitus," J Biomed Eng, vol. 14, pp. 235-242, 1992. 